\title{
Band-stop filter with suppression of requested number of spurious stopbands
}

\author{
DUSAN A. NESIC ${ }^{1}$ AND BRANKO M. KOLUNDZIJA ${ }^{2}$
}

\begin{abstract}
Design method for band-stop filters (BSFs) that suppress a requested number of spurious bandstops and reduce ripples in the passbands below $\sim_{1} \mathrm{~dB}$ is proposed. BSF is designed in a form of a cascade of cells, each consisting of steps of equal electrical length, where the number of steps is used to control the number of suppressed spurious bandstops. Analytical formulas are developed that enable initial design of BSF for a given central frequency, depth, and bandwidth of the stopband. Varying the minimum characteristic impedances of initial cells, through an optimization using circuit simulation, the ripples in passbands are reduced below $\sim_{1} \mathrm{~dB}$. Using the proposed theory, three filters in microstrip technology, with suppression of 3, 5, and 7 spurious stopbands respectively, were designed, fabricated, and measured. Good agreement between simulated and measured results has been observed. The proposed design can be recommended for filters having broad stop bandwidths, between 40 and $100 \%$.
\end{abstract}

Keywords: Band-stop filter, Suppression of spurious stop-bands, Microstrip

Received 17 February 2016; Revised 17 August 2016; Accepted 22 August 2016; first published online 21 September 2016

\section{INTRDDUCTIDN}

Bandstop filters (BSFs) realized in microstrip technology represent important building blocks in modern $\mathrm{RF} /$ microwave communication systems. Conventional all-transmission line BSFs have spurious stopbands repeated at odd multiples of the fundamental stopband center frequency, $f_{\mathrm{o}}[1]$. In practice, however, much wider upper passbands may be required.

The method most frequently used to extend the upper passbands of BSFs is to shift the higher-order resonances higher in frequency utilizing parallel coupled line, stepped-impedance or lumped element-loaded resonators [2-8]. Using these techniques upper passbands extended up to $6 f_{\mathrm{o}}$ are reported for narrow band and moderate band BSFs.

Alternatively, the upper passbands can be extended by suppressing the higher-order resonances. One way to suppress spurious resonances is based on using a constructive interference technique [9]. Using this technique the upper passband extended up to $9 f_{\mathrm{o}}$ is reported for noch filters. Another way to suppress the spurious resonances is based on application of periodically nonuniform microstrip lines.

In general, periodically nonuniform microstrip lines exhibit bandstops repeated at multiples of $f_{\mathrm{o}}$ [10-12]. Sinusoidal and triangular patterns etched in the ground plane of a microstrip line can substantially suppress the spurious bandstop at $2 f_{\mathrm{o}}[13]$. A sinusoidal variation of the width of the microstrip line $[14,15]$, results in similar suppression. If

${ }^{1}$ University of Belgrade, IHTM-CMT, Njegoseva 12, 11000 Belgrade, Serbia. Phone: 381-11-2630757

${ }^{2}$ University of Belgrade, School of Electrical Engineering, Bul. King Alexander 73, Serbia. Phone: 381-11-3218329; Fax: 381-11-3288681

Corresponding author:

D.A. Nesic

Email: nesicad@nanosys.ihtm.bg.ac.rs sinusoidal variation is applied to microstrip characteristic impedance, spurious stopbands around $2 f_{\mathrm{o}}$ and $3 f_{\mathrm{o}}$ can be fully suppressed [16]. Simulations and measurements above $3 f_{\mathrm{o}}$, which are not presented in the paper, show that higherorder stopbands are only partially suppressed. Starting from the coupled mode theory, an analytical solution for periodically nonuniform transmission lines that are free of spurious stopbands is proposed [17-19]. The validity of the theory is demonstrated on the example of moderate band BSF with measured results showing full suppression of spurious bandstops at $2 f_{\mathrm{o}}$ and $3 f_{\mathrm{o}}$.

All of the above mentioned BSFs, which are based on periodically nonuniform microstrip lines, suffer from relatively deep ripples in the passbands. The depth of these ripples increases up to $\sim_{5} \mathrm{~dB}$ near the stopband. It is shown that in the case of a cascade of four trapezoidally shaped cells, which eliminates spurious stopbands at $2 f_{\mathrm{o}}$ and $3 f_{\mathrm{o}}$, the depth of these ripples can be reduced down to $\sim_{1} \mathrm{~dB}$ by simple tapering, i.e. narrowing outer cells and widening the inner cells [20]. Similar technique for reducing ripples is used in the case of BSFs made of cells consisting of cascades of uniform transmission lines (steps) $[21,22]$. In the case where cell consists of six steps, suppression of three spurious bands is confirmed by measurement, while in the case where cell consists of nine steps, the suppression of six spurious bands is confirmed by EM simulation.

The paper proposes a design method for BSFs that suppresses a requested number of spurious bandstops and reduces ripples in the passbands below $\sim_{1} \mathrm{~dB}$. On one hand, the design method represents the generalization of procedures presented in $[21,22]$. On the other hand, it leans on the theory of periodically nonuniform transmission lines that are free of spurious stopbands [17-19]. Construction of unit cell is described in Section II. Design method is proposed 
in Section III. Three-dimensional (3D) EM simulation, fabrication, and measurement of three typical filters are presented in Section IV.

\section{UNIT CELL}

Consider $1 \mathrm{D}$ periodic structure in the form of infinite cascade of identical cells, where each cell represents a cascade of $n$ steps without losses, of equal angular length $\theta$, and of different characteristic impedances, $Z_{i}, i=1, \ldots, n$, as shown in Fig. 1 . (Adopting equal angular length for all steps decreases the flexibility of a unit cell, but enables derivation of analytical design formulas.)

Following the general theory of periodic structures [23] the cell can be characterized in two ways: (1) with ABCD matrix, and (2) with its physical length $l$ and equivalent propagation coefficient $\gamma=\alpha+j \beta$, where $\alpha$ is equivalent attenuation coefficient and $\beta$ is equivalent phase coefficient. In the case $|(A+D) / 2| \leq 1$, the wave propagates along infinite periodic structure without attenuation $(\alpha=0, \beta \neq 0)$, while in the case $|(A+D) / 2|>1$ the wave attenuates along the structure $(\alpha \neq 0, \beta=0)$. If infinite structure is truncated, the attenuation in stopbands is limited by number of cells, while propagation in passbands is disturbed due to reflection in planes of truncation. In order to minimize the reflections, the Bloch impedance in planes of truncation should be as close as possible to reference impedance $Z_{\mathrm{o}}$ in whole passband of interest. The first step in designing the BSF is to determine the unit cell that provides desired stopbands and the Bloch impedance as close as possible to $Z_{\mathrm{o}}$ in passbands.

ABCD matrix of the cell having $i$ steps can be represented as a product of ABCD matrix of the cell having $i-1$ steps and ABCD matrix of $i$ th step as

$$
\left[\begin{array}{cc}
A_{i} & B_{i} \\
C_{i} & D_{i}
\end{array}\right]=\left[\begin{array}{ll}
A_{i-1} & B_{i-1} \\
C_{i-1} & D_{i-1}
\end{array}\right] \cdot\left[\begin{array}{cc}
\cos \theta & j Z_{i} \sin \theta \\
\frac{j}{Z_{i}} \sin \theta & \cos \theta
\end{array}\right]
$$

$A$ and $D$ parameters of unit cell are obtained after (1) is repeated $n_{-} 1$ times $\left(A=A_{n}\right.$ and $\left.D=D_{n}\right)$. In general case $(A+D) / 2$ is obtained in the form of polynomial in terms of $\cos \theta$

$$
\frac{A_{n}+D_{n}}{2}=\sum_{k=0}^{[n / 2]} p_{k}^{(n)}(\cos \theta)^{n-2 k}
$$

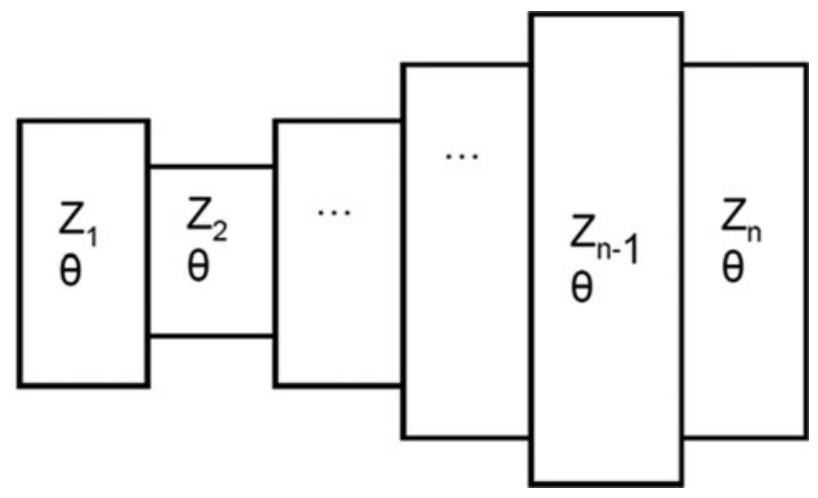

Fig. 1. Unit cell: $n$ steps of equal angular length $\theta$ and different characteristic impedances $Z_{k}$ where $[n / 2]$ represents integer part of $n / 2$ and coefficients $p_{k}^{(n)}$ depends only on characteristic impedances $Z_{i}, i=1, \ldots, n$. In the following text this polynomial will be referred to as dispersion polynomial.

Dispersion polynomial is a periodic function in $\theta$, with period of $2 \pi$. Since it is either even or odd function with respect to $\theta=0$, it is sufficient to explore the properties of this polynomial in range $0 \leq \theta \leq \pi$. Using (1), it is easy to show that at boundaries of the range the polynomial always has values \pm 1 , which means that $\theta=0$ and $\theta=\pi$ always belong to the passband. In a general case, inside the range the polynomial may have up to $n$ zeros and $n-1$ extrema. If magnitudes of all extrema are $>_{1}$, there are $n-1$ stopbands in the range. Finally, having in mind that $\cos (\pi-\theta)=-\cos (\theta)$, both, the stopbands and passbands, are symmetrical with respect to $\theta=\pi / 2$.

By numerical experiment it is found that random choice of $n$ characteristic impedances of a unit cell most often results in all $n-1$ stopbands $[21,22]$, with the centers of stopbands, $\theta_{k}$, $k=1, \ldots, n-1$, almost uniformly distributed. It is also found that, for specific values of characteristic impedances, one or more pairs of stopbands symmetrical to $\theta=\pi / 2$ can be eliminated. Since the 1 st stopband is coupled with the $(n-1)$-st stopband, the maximum number of stopbands that can be eliminated is

$$
p=n-3
$$

The first stopband is located at $\theta_{1}=\pi / n$, and the total electrical length of the cell is $n \theta_{1}=\pi$.

To eliminate these stopbands we apply the multi-minima optimization method, which enables finding a set of local minima in a single run [24]. The characteristic impedance values of all steps are varied between given $Z_{\text {min }}$ and $Z_{\text {max }}$. For each set of characteristic impedances, the extrema of dispersion polynomial are determined, excluding the extrema related to the first and the last stopband. The functional that is minimized by optimization procedure is obtained as mean square value of extrema whose magnitudes are $>_{1}$. Theoretically, the solution is found when the value of the functional decreases to one. In practice we consider that the solution is found when value of functional decreases below 1.001 .

At the beginning we varied all impedances independently. Common for all solution was that width of the stopband depends on ratio $Z_{\text {max }} / Z_{\text {min }}$. We also found that Bloch impedance in the whole passband is closest to

$$
Z_{\mathrm{o}}=\sqrt{Z_{\max } Z_{\min }}
$$

if characteristic impedances in the first half of cell are lower than or equal to $Z_{0}$, while in the second half they are greater than or equal to $Z_{\mathrm{o}}$. When these constraints are applied, it has been found that characteristic impedances in the first and second half of cell are related as

$$
Z_{k} Z_{n-k+1}=Z_{0}^{2}
$$

In particular, for odd $n$, according to (5), the impedance of central step is $Z_{\mathrm{o}}$. It has also been shown that minimum characteristic impedance occurred at 2nd step for $n=5,6,7$, and 
8 , 3 rd step for $n=9,10,11$, and 12, etc. Thus, the number of independently varied impedances was more than halved.

As a result of imposing the constraints as above, and fixing the step with minimum characteristic impedance to given $Z_{\text {min }}$, a practically unique solution has been obtained for each $n$. "Practically" means that in all optimizations repeated for the same $Z_{\text {min }}$ and $n$, different solutions for characteristic impedances differed on the 4 th significant digit, or higher. Having this in mind we systematically changed $Z_{\text {min }} / Z_{\mathrm{o}}$ from 0.2 to 1.0 for $n=5,6,7,8,9$, and 10. The results obtained in this manner are interpolated using 2 nd order polynomials. The corresponding analytical expressions are given in Table 1. These expressions together with equation (5) completely define unit cells.

In order to illustrate the proposed theory let us consider BSF in the form of a cascade of four identical unit cells. Electrical length of unit cell is set to $\pi$ at frequency of $3 \mathrm{GHz}$, so that center frequency of the 1 st stopband falls at $f_{\mathrm{o}}=3 \mathrm{GHz}$. Minimal characteristic impedances of cells consisting of $n=5,6,7,8,9$, and 10 steps are set to $Z_{\text {min }}=$ $25.725 .1,25.8,26.8,25.6$, and $25.6 \Omega$, respectively, for the nominal impedance of ports set to $Z_{\mathrm{o}}=50 \Omega$, so that minimum $S_{21}$ is adjusted to approximately $-30 \mathrm{~dB}$. Figure 2 shows $S_{21}$-parameter versus frequency for various number of steps of unit cell, $n=5,6,7,8,9$, and 10. It is seen that for $n$ steps the number of suppressed higher bandstops is $n-3$, as predicted.

Generally, it is shown that by increasing number of steps the shape of cascade of unit cell approaches the periodic nonuniform transmission line that does not have any spurious stopband $[17-19]$. It means that solution proposed in this paper represents a kind of step approximation of such nonuniform line. Another way to obtain step approximation of the line is to uniformly subdivide the line into $n$ segments and replace each segment with the step whose characteristic impedance corresponds to the center of the original segment. Starting from formula for characteristic impedance of nonuniform line from [17-19], the characteristic impedances in step approximation can be written as

$$
\begin{aligned}
Z_{k} & =Z\left(x_{k}\right)=Z_{0}\left(\frac{Z_{\text {min }}}{Z_{\text {max }}}\right)^{1 / 2 \sin \left(2 \pi / l x_{k}\right)} x_{k}=\frac{2 k-1}{n} l k \\
& =1, \ldots, n .
\end{aligned}
$$

It is shown that values for $Z_{k}, k=1, \ldots, n$, obtained by equation (6) are similar to those given in Table 1 , and the similarity is improved with increasing $n$. If these values are applied to obtain results for the above defined BSF, results similar to those presented in Fig. 2 are obtained. Exception is that minimum $S_{21}$ value is increased (from $-30 \mathrm{~dB}$ ) for 2.1, 1.4, $0.9,0.6,0.4$, and $0.3 \mathrm{dBs}$ for $n=5,6,7,8,9$, and 10 steps, respectively. It is obvious that accuracy of equation (6) improves for higher $n$. Accordingly, Table 1 contains formulas up to $n=10$.

\section{DESIGN DF BSF}

It is shown that stopband of a truncated periodic nonuniform transmission line, which is free of spurious stopbands, and the first stopband of its step approximation practically coincide. Starting from formulas given in $[17-19]$, the minimum value of $S_{21}$ of the stopband can be expressed as $\left|S_{21}\right|_{\text {min }}=\operatorname{sech}\left[m \pi / 4 \ln \left(Z_{\text {min }} / Z_{\text {max }}\right)\right]$, where $m$ is the number of unit cells. For $m>2$, and using (4) the approximation of $\left|S_{21}\right|_{\text {min }}$ is obtained in the form

$$
\left|S_{21}\right|_{\min } \cong 2\left(\frac{Z_{\text {min }}}{Z_{\text {max }}}\right)^{m \pi / 4}=2\left(\frac{Z_{\text {min }}}{Z_{\mathrm{o}}}\right)^{m \pi / 2}
$$

Starting from formulas given in [17-19], the bandwidth of a stopband measured between its zeros can be expressed as $\mathrm{BW}=f_{\mathrm{o}} \sqrt{\left[\ln \left(Z_{\max } / Z_{\min }\right) / 2\right]^{2}+(2 / m)^{2}}$. Using (7) the expression for bandwidth is obtained in an approximate form as:

$$
\mathrm{BW}=f_{\mathrm{o}} \frac{2}{m} \sqrt{1+\left(\frac{1}{\pi} \ln \frac{2}{\left|S_{21}\right|_{\min }}\right)^{2}} .
$$

The design procedure based on formulas (3)-(8) and Table 1 is established as follows. Input data for a design are: central frequency of the stopband, $f_{\mathrm{o}}$, bandwidth of the stopband, BW, the maximum permissible value of $\left|S_{21}\right|_{\text {min }}$, the nominal impedance $Z_{\mathrm{o}}$, and number of spurious bandstops that should be suppressed, $p$. The basic output data for the design are: number of steps, $n$, number of unit cells, $m$, and minimum characteristic impedance $Z_{\text {min }}$. These data are determined using following steps:

1) for given $p$ the number of steps $n$ is determined from (3);

\begin{tabular}{|c|c|}
\hline$n$ & Analytical formulas for characteristic impedances $Z_{k}, k=1,[n / 2]$ \\
\hline $\begin{array}{l}5 \\
6\end{array}$ & $\begin{array}{l}0.0056 \cdot Z_{0}+0.3195 \cdot Z_{1}+0.6955 \cdot \frac{Z_{1}^{2}}{Z_{0}}=Z_{2}=Z_{\min } \\
Z_{1}=Z_{3}=\sqrt{Z_{\min } Z_{0}} Z_{2}=Z_{\min }\end{array}$ \\
\hline 7 & $Z_{1}=0.3466 \cdot Z_{0}+0.9063 \cdot Z_{\min }-0.2610 \cdot \frac{Z_{\min }^{2}}{Z_{0}} Z_{2}=Z_{\min } Z_{3}=0.0824 \cdot Z_{0}+1.0488 \cdot Z_{\min }-0.1340 \cdot \frac{Z_{\min }^{2}}{Z_{0}}$ \\
\hline 8 & $Z_{1}=Z_{4}=0.3786 \cdot Z_{0}+0.8750 \cdot Z_{\min }-0.2610 \cdot \frac{Z_{\min }^{2}}{Z_{0}} Z_{2}=Z_{3}=Z_{\min }$ \\
\hline 9 & $\begin{array}{l}Z_{1}=0.4565 \cdot Z_{0}+0.7821 \cdot Z_{\min }-0.2450 \cdot \frac{Z_{\min }^{2}}{Z_{0}} Z_{2}=0.0519 \cdot Z_{0}+1.0158 \cdot Z_{\min }-0.0655 \cdot \frac{Z_{\text {min }}}{Z_{0}} Z_{3}=Z_{\text {min }} \\
Z_{4}=0.1639 \cdot Z_{0}+1.0628 \cdot Z_{\min }-0.2345 \cdot \frac{Z_{\text {min }}^{2}}{Z}\end{array}$ \\
\hline 10 & $Z_{1}=Z_{5}=Z_{2} \sqrt{\frac{Z_{0}}{Z_{\min }}} Z_{2}=Z_{4}=0.0777 \cdot Z_{0}+1.0558 \cdot Z_{\min }-0.1370 \cdot \frac{Z_{\min }^{2}}{Z_{0}} Z_{3}=Z_{\min }$ \\
\hline
\end{tabular}

2) for given $f_{\mathrm{o}}, \mathrm{BW}$, and the maximum permissible $\left|S_{21}\right|_{\text {min }}$ the number of unit cells $m$ is determined from (8); as a result is

Table 1. Analytical expressions for characteristic impedances of unit cell in terms of $Z_{\text {min }}$. 

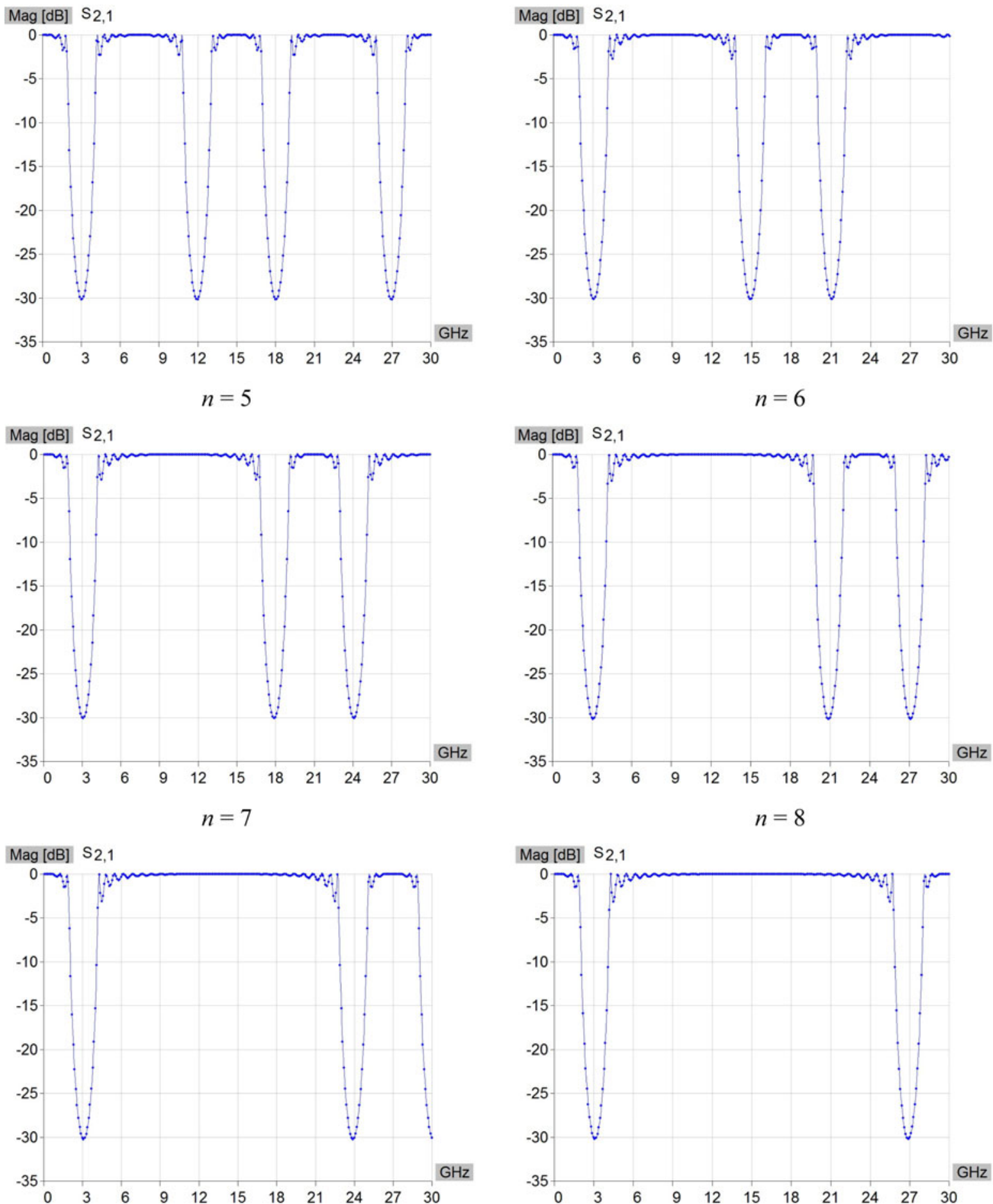

Fig. 2. $S_{21}$-parameter versus frequency for BSF made of four identical unit cells, for various number of unit cell steps, $n=5,6,7,8,9$, and 10 .

generally not an integer, the final value of $m$ is adopted to be a value of the first higher integer;

3) for given $f_{\mathrm{o}}, \mathrm{BW}$, and $m$ calculated from previous step, $\left|S_{21}\right|_{\min }$ is determined from (8); value of such obtained $\left|S_{21}\right|_{\text {min }}$ is higher than given maximum allowed value;

4) from the given $Z_{0}$ and values for $m$ and $\left|S_{21}\right|_{\text {min }}$ as calculated above, the value for minimum characteristic impedance $Z_{\text {min }}$ is determined.

Once we know these data, characteristic impedances of all steps are determined using equation (5) and Table 1 for $n \leq$
10 and equation (6) for $n>10$. In particular, this design procedure can be applied to a unit cell in the form of the segment of periodically nonuniform transmission line free of spurious stopbands [19], which length is equal to one period of this line. Note that such a cell is obtained from the unit cell proposed in this paper when number of steps $n$ tends to infinity.

Using the unit cell of arbitrary $n$ instead of cell, which $n$ tends to infinity gives additional flexibility in choosing which harmonics should be suppressed. For example, suppression of the 1st and the $k$ th harmonic can be performed using a cell of $n=k+1$ steps. Besides that, suppression of 

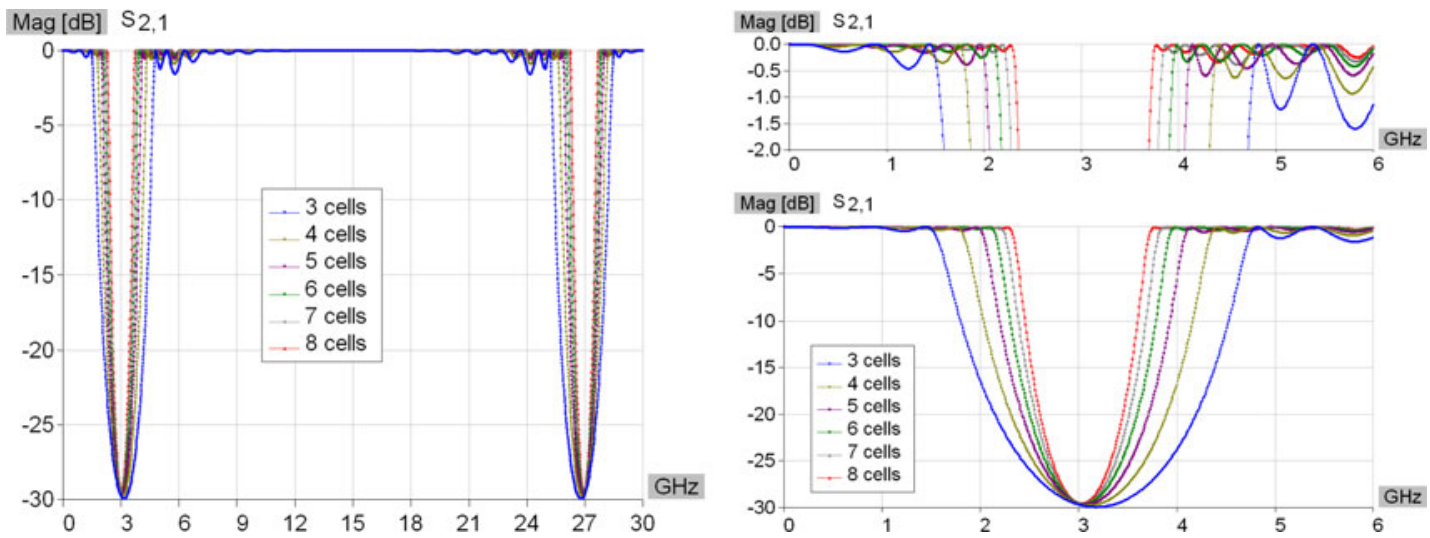

Fig. 3. $S_{21}$-parameter versus frequency for optimized BSFs made of various number of cells, $m=3,4,5,6$, 7 , and 8 , where each cell is made of 10 steps.

a given number $p$ of higher spurious stopbands can be performed using unit cell having any number of steps $n>p+$ 2, which gives additional flexibility in choosing the geometry of the filter. For example, it is often easier to fabricate the cascade of steps then continuously nonuniform transmission line.

Major drawback of such designed BSF is appearance of ripples, up to $5 \mathrm{dBs}$ deep, around the first stopband. Generally, these ripples can be reduced by optimizing values of characteristic impedances of all $n$ steps for each of $m$ cells. In addition, such optimization should preserve initial BW and $\left|S_{21}\right|_{\text {min }}$. However, an optimization based on so many variables is extremely time-consuming and can easily fail to significantly improve the initial solution. It is found that such optimization is much more effective if only values of minimum characteristic impedances of all cells are varied, i.e., $Z_{\text {min }}^{(i)}, i=1, . ., m$. Note that by varying minimum characteristic impedance of the $i$ th cell, $Z_{m i n}^{(i)}$, the characteristic impedances of all other steps in the cell are automatically varied according to either formula (5) and Table 1, or formula (6). Therefore, the number of optimization variables is reduced to the number of cells $m$. It is also found that cells positioned symmetrically to the center of the filter should have equal values of minimum characteristic impedances

$$
Z_{\text {min }}^{(m-i+1)}=Z_{\text {min }}^{(i)}
$$

Thus the number of optimization variables $m$ is practically halved. Finally, we found that initial BW and $\left|S_{21}\right|_{\text {min }}$ are

Table 2. Optimized minimum characteristic impedances $Z_{\min }^{(i)}$ for filters made of various number of unit cells $m$, with each unit cell comprises $n=10$ steps. The maximum ripple $S_{21}$ is given before and after the optimization. (Note that $[(m+1) / 2]$ represents integer part of $(m+1) / 2$.)

\begin{tabular}{lllll}
\hline & & \multicolumn{2}{c}{ Maximum ripple (dB) } \\
\cline { 3 - 4 } $\boldsymbol{m}$ & $Z_{\text {min }}^{(i)}, i=1, \ldots,\left[\frac{m+1}{2}\right][\Omega]$ & \multicolumn{1}{c}{$\begin{array}{c}\text { Before } \\
\text { optimization }\end{array}$} & $\begin{array}{c}\text { After } \\
\text { optimization }\end{array}$ \\
\hline 3 & 21.918 .6 & -3.17 & -1.60 \\
4 & 28.323 .5 & -3.09 & -0.94 \\
5 & 32.528 .026 .8 & -3.01 & -0.59 \\
6 & 36.231 .429 .4 & -2.95 & -0.42 \\
7 & 38.734 .731 .631 .2 & -2.82 & -0.32 \\
8 & 40.437 .333 .533 .2 & -2.86 & -0.34 \\
\hline
\end{tabular}

practically preserved if geometric mean of all minimum characteristic impedances is also preserved, i.e.

$$
\sqrt[m]{\prod_{i=1}^{m} Z_{\min }^{(i)}}=Z_{\min } .
$$

In the case of $m=3$ and $m=4$ cells, there is only one optimization parameter $Z_{\text {min }}^{(1)}$. In the case of $m=5$ and $m=6$ cells, there is only two optimization parameter $Z_{\text {min }}^{(1)}$ and $Z_{\text {min }}^{(2)}$, etc.

In order to illustrate the effectiveness of the procedure for suppression of ripples in the vicinity of stopbands as explained, let us consider BSF filters made of 3, 4, 5, 6, 7, and 8 cells, each cell made of 10 steps, whose minimum value of $S_{21}$ is equal to $-30 \mathrm{~dB}$ with respect to the nominal impedance of ports set to $Z_{\mathrm{o}}=50 \Omega$. After the optimization, minimum characteristic impedances are obtained as given in Table 2. Figure 3 shows $S_{21}$-parameter for optimized filters. The values of the maximum ripple $S_{21}$ before and after the optimization are also given in Table 2. Before the optimization these values are approximately $-3 \mathrm{~dB}$, while after the optimization these values are $>-1 \mathrm{~dB}$.

It is also seen from Fig. 3 that by reducing width of the bandstop a number of cells must be increased to maintain given $S_{21}$. Consequently, the proposed design is less suitable for very narrow bandstops, as a relatively high number of cells is required in this case. On the other hand, by increasing width of the bandstop, the ripples in its vicinity become more pronounced. Having all above in mind, the proposed design can be recommended for filters having broad stopband widths, between 40 and $100 \%$ with respect to the central bandstop frequency. It also means that such design is complementary to other designs referenced in this paper. Namely, designs from references [2, 7, and 9] enable narrow stop bandwidths, up to $10 \%$, while designs from references, [3-6] and [8] enable stop bandwidths up to $40 \%$.

Table 3. Optimized values of minimum impedances of unit cells, for filter made of four cells, where each cell consists of $n$ steps.

\begin{tabular}{lccc}
\hline $\boldsymbol{n}$ & $\mathbf{6}$ & $\mathbf{8}$ & $\mathbf{1 0}$ \\
\hline$Z_{\min }^{(1)}=Z_{\min }^{(4)} \Omega$ & 27.60 & 29.27 & 27.90 \\
$Z_{\min }^{(2)}=Z_{\min }^{(3)}[\Omega]$ & 22.82 & 24.53 & 23.49 \\
\hline
\end{tabular}




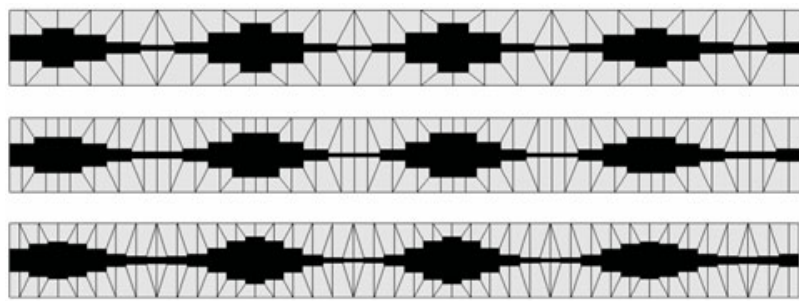

(a)

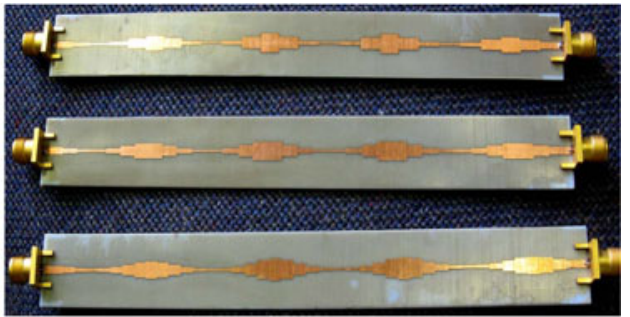

(b)

Fig. 4. Optimized BSFs made of four cells, each cell of $n=6,8$ and 10 steps: (a) geometrical models for 3 D EM simulation, (b) photo of the prototypes

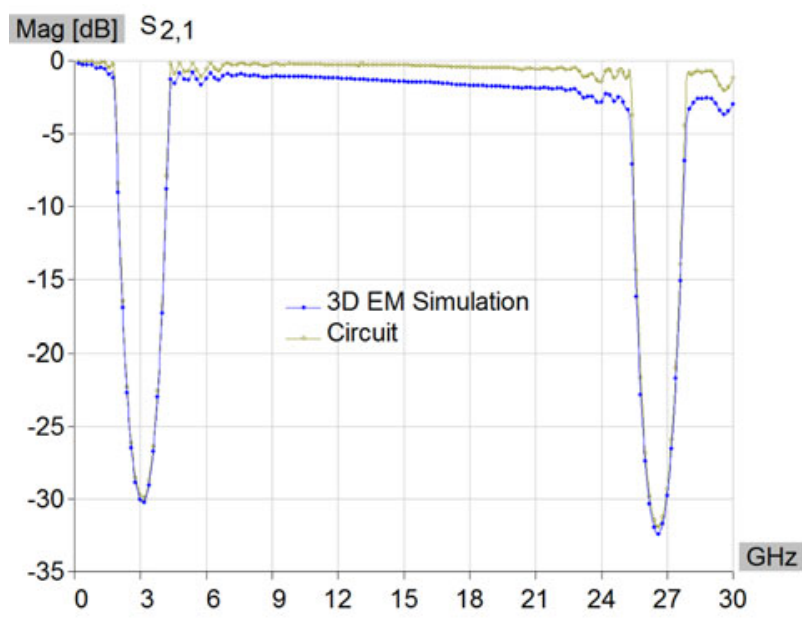

(a)

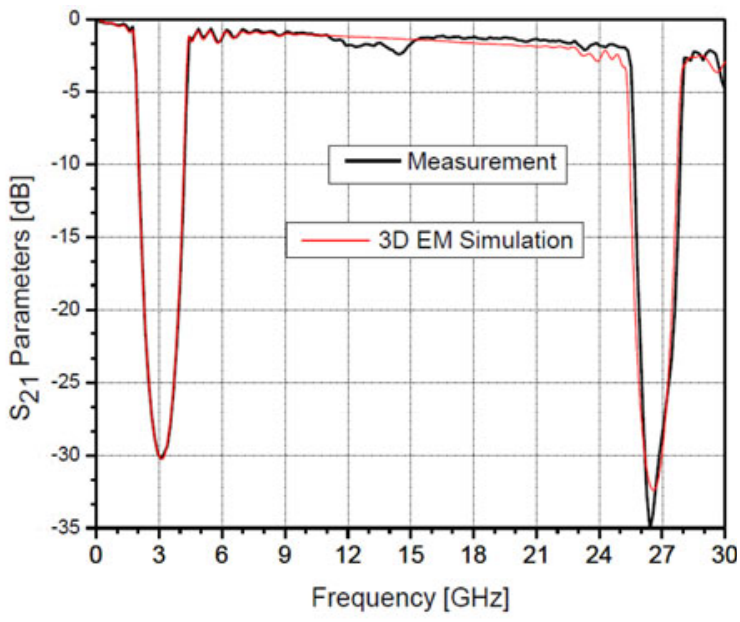

(b)

Fig. 5. $S_{21}$ parameters of BSF made of four cells, each consisting of $n=10$ steps: (a) comparison of circuit and $3 \mathrm{D}$ EM simulation, and (b) measurement and $3 \mathrm{D}$ EM simulation

\section{3DEM SIMULATIDN FABRICATIDN, AND MEASUREMENT}

Three BSFs are designed starting from the following input data: $f_{\mathrm{o}}=3 \mathrm{GHz}, \mathrm{BW}=2.5 \mathrm{GHz},\left|S_{21}\right|_{\min }=-30 \mathrm{~dB}, Z_{\mathrm{o}}=$

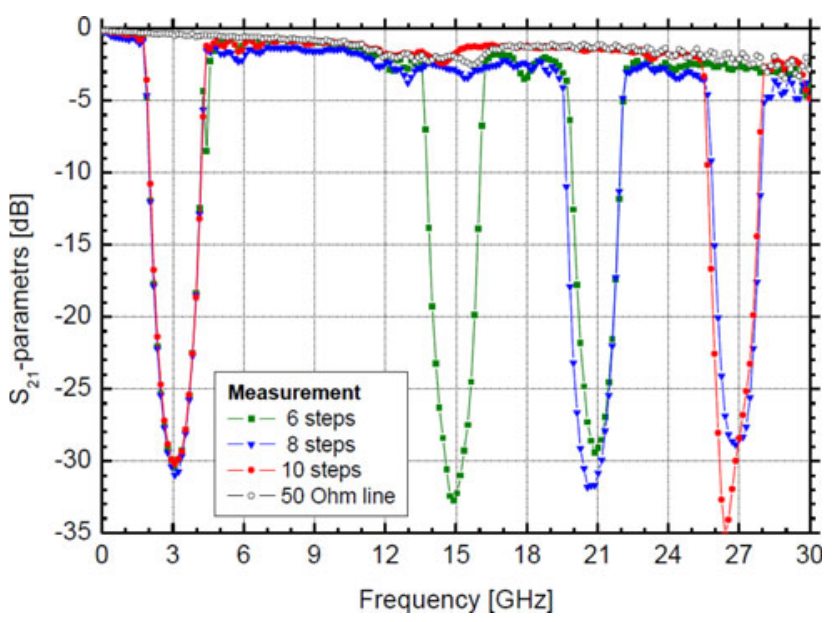

Fig. 6. Measured $S_{21}$-parameter for nominal $50 \Omega$ line and three BSFs made of four cells, each cell of $n=6,8$ and 10 steps.
$50 \Omega$, and number of spurious stopbands that should be suppressed, $p=3,5$, and 7 . Note that the total electrical length of each cell $\pi$ corresponds to $\lambda_{g} / 2$, where $\lambda_{g}$ is wavelength in microstrip line. According to the proposed procedure, the numbers of steps of unit cells of these filters are $n=6,8$, and 10, respectively, the number of cells is $m=4$, and the corresponding optimized values of minimum impedances for these cells are given in Table 3 .

These BSFs are realized in microstrip technology, on the CuFlon substrate of relative permittivity $\varepsilon_{r}=2.1$, loss tangent $\tan \delta=0.0004$, thickness $h=0.508 \mathrm{~mm}$, metallization thickness $t=17 \mu \mathrm{m}$, and metallization conductivity $\sigma=58 \mathrm{MS} / \mathrm{m}$. The properties of the CuFlon substrate are used to determine the physical widths and lengths of the steps so that their characteristic impedances and electrical lengths correspond to the design values at the central frequency of the stopband. Since the major part of the current flow in microstrip lines is placed near their edges, and the edge of the step discontinuity belongs to the wider microstrip line, the total electrical length of the current path in the wider line is slightly longer than its initially determined electrical length. In order to adjust the electrical lengths of this current path to the design value it is found by numerical experiment that the wider line should be shortened for about $5 \%$ of the difference in width between the wider and the narrower line. Geometrical models of filters, as analyzed in WIPL-D Pro 3D EM solver [25], are shown in Fig. 4(a). For 


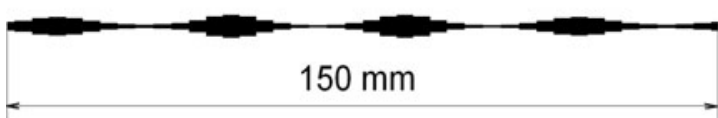

(a)

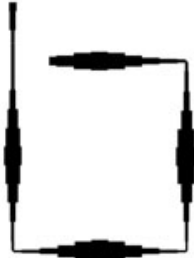

(b)

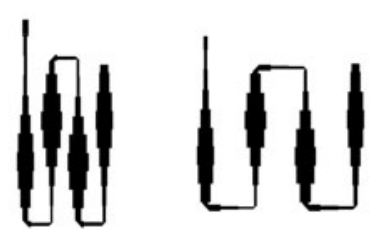

(c)

(d)

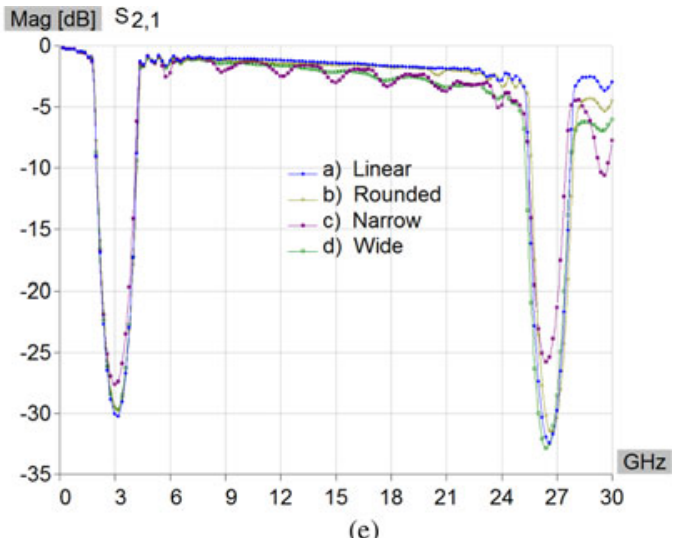

(e)

Fig. 7. Footprints of filters: (a) linear, (b) rounded, (c) narrow meandered, and (d) wide meandered filter; (e) corresponding $S_{21}$-parameters obtained by 3 D EM simulation.

simulation purposes, to take into account the surface roughness of metallization, the conductivity has been set to $\sigma=$ $18 \mathrm{MS} / \mathrm{m}$. The filters are fabricated and measured using the extended-frequency SMA connectors at both ends. Photos of the fabricated prototypes are shown in Fig. 4(b). (The results for filter with $n=6$ steps have been presented in [21], and are repeated here for the sake of completeness.) The total length of these filters is $\sim 150 \mathrm{~mm}$. It is shown that the maximum filter dimension can be reduced up to 4 times by bending or meandering without significant degradation of its performance (see Appendix).

Figure 5 shows results for $S_{21}$-parameter of the filter with unit cells made of $n=10$ steps. Unlike $3 \mathrm{D}$ EM simulation, the circuit simulation does not take into account losses. The results obtained using EM simulations are in excellent agreement with measurement.

Figure 6 shows measured $S_{21}$-parameter for all three BSFs, compared with measured $S_{21}$-parameter of microstrip line of nominal characteristic impedance $Z_{\mathrm{o}}=50 \Omega$. It can be concluded that in the passbands the filters practically behave as a $50 \Omega$ line with negligible extra losses. A value of the insertion loss of the filters is comparable with the published results of similar filters.

\section{CDNCLUSION}

Design method for a BSF that suppresses a requested number of spurious bandstops is proposed. Initially, BSF is set in the form of a cascade of identical cells, where each cell consists of steps (uniform transmission lines) of equal electrical length. The characteristic impedances of the cell steps are uniquely determined by the ratio of maximum and minimum characteristic impedance of the cell, where the geometric mean of these two impedances is equal to the nominal impedance. A number of suppressed spurious bandstops $p$ is directly related to the number of steps in unit cell $n$, i.e., $p=n-3$. Bandstop width of the filter and attenuation at the central frequency of the stopband are related to the ratio of maximum and minimum characteristic impedance of the cell and the number of cells. Varying the minimum characteristic impedances of all cells, keeping the geometric mean of the impedances at the initial value, the ripples in passbands can be reduced below $\sim_{1} \mathrm{~dB}$.
The number of steps in a cell can go up to infinity, in which case this cell represents a segment of periodically nonuniform transmission line free of spurious stopbands [19], whose length is equal to one period of this line. Using the unit cell of arbitrary $n$ instead of cell whose $n$ tends to infinity gives additional flexibility in choosing harmonics suppression (e.g., the 1st and the $k$ th harmonic can be suppressed using a cell of $n=k+1$ steps, or suppression of a given number $p$ of higher spurious stopbands can be performed using unit cell having any number of steps $n>p+2$ ). Besides that, it is often easier to fabricate the cascade of steps then continuously nonuniform transmission line.

Using the proposed theory, three filters in microstrip technology suppressing 3, 5, and 7 spurious stopbands respectively, are designed, fabricated, and measured. Excellent agreement between simulated and measured results is observed, confirming that proposed method can be efficiently used for design of a BSF that suppresses a requested number of spurious bandstops

The proposed design can be recommended for filters having broad stop bandwidths, between 40 and 100\% with respect to the central bandstop frequency.

\section{ACKNDWLEDGEMENTS}

This work was funded by Serbian Ministry of Education and Science, project TR 32005 .

\section{REFERENCES}

[1] Hong, J.-S.: Microstrip filters for RF/Microwave Applications, 2nd ed., John Wiley and Sons, Hoboken, NJ, 2011.

[2] Snyder, R.V.; Shin, S.: Parallel Coupled Line Notch Filter with Wide Spurious-Free Passbands, in IEEE MTT-S Int. Microwave Symp. Digest, Long Beach, 12-17 June 2005, 253-256.

[3] Levy, R.; Snyder, R.V.; Shin, S.: Bandstop filters with extended upper passbands. IEEE Trans. Microw. Theory Tech., 54 (6) (2006), 25032515 .

[4] Fathelbab, W.M.; Steer, M.B.: Design of bandstop filters utilising circuit prototypes. IET Microw. Antennas Propag., 1 (2) (2007), $523-526$. 
[5] Alkanhal, M.A.S.: Compact bandstop filters with extended upper passbands. Act. Passive Electron. Compon., 2008, Article ID 356049, 2008.

[6] Ma, K.; Jayasuriya, R.M.; Chan, K.T.: High Performance Bandstop Filter Design and Investigation Using Physical Model for WiMAX Measurement Equipment, IEEE MTT-S Int. Microwave Symp. Digest, Atlanta, 15-20 June 2008, 1247-1250.

[7] Fathelbab, W.M.: Two novel classes of band-reject filters realizing broad upper pass bandwidth-synthesis and design. IEEE Trans. Microw. Theory Tech., 59 (2) (2011), 250-259.

[8] Wu, Y.; Liu, Y.: A coupled-line band-stop filter with three-section transmission line stubs and wide upper pass-band performance. Prog. Electromagn. Res., 119 (2011), 407-421.

[9] Guyette, A.C.: Design of Fixed- and Varactor-Tuned Bandstop Filters with Spurious Suppression, in Proc. of the 4oth European Microwave Conf., Paris, 28-30 September 2010, 288-291.

[10] Glandorf, F.J.; Wolff, I.: A spectral-domain analysis of periodically nonuniform microstrip line. IEEE Trans. Microw. Theory Tech., 35 (3) (1987), 336-343.

[11] Radisic, V.; Qian, Y.; Itoh, T.: PBG structures for microstrip circuits. Microw. Rev., 4 (2) (1997), 8-11.

[12] Rumsey, I.; Piket-May, M.; Kelly, P.K.: Photonic bandgap structures used as filters in microstrip circuits. IEEE Microw. Guid. Wave Lett., 8 (10) (1998), 336-338.

[13] Lopetegi, T.; Laso, M.A.G.; Erro, M.J.; Benito, D.; Garde, M.J.; Falcone, F. et al.: Novel photonic bandgap microstrip structures using network topology. Microw. Opt. Technol. Lett., 25 (1) (2000), 33-36.

[14] Nair, N.V.; Mallick, K.: An analysis of a width-modulated microstrip periodic structure. IEEE Trans. Microw. Theory Tech., 32 (2) (1984), 200-204.

[15] Lopetegi, T.; Laso, M.A.G.; Hernandez, J.; Bacaicoa, M.; Benito, D.; Garde, M.J. et al.: New microstrip "wiggly-line" filters with spurious passband suppression. IEEE Trans. Microw. Theory Tech., 49 (9) (2001), 1593-1598.

[16] Nesic, D.; Nesic, A.: Bandstop microstrip PBG filter with sinusoidal variation of the characteristic impedance and without etching in the ground plane. Microw. Opt. Technol. Lett., 29 (6) (2001), 418-420.

[17] Lopetegi, T.; Laso, M.A.G.; Erro, M.J.; Sorolla, M.; Thumm, M.: Analysis and design of periodic structures for microstrip lines by using the coupled mode theory. IEEE Microw. Wirel. Compon. Lett., 12 (11) (2002), 441-443.

[18] Arnedo, I.; Schwartz, J.D.; Arregui, I.; Laso, M.A.G.; Plant, D.V.; Azaña, J. et al.: Analytical solution for the design of planar EBG structures with spurious-free frequency response, in Proc. of European Microwave Conf., 29 September-1 October 2009, 12991302.

[19] Arnedo, I.; Chudzik, M.; Schwartz, J.D.; Arregui, I.; Lujambio, A.; Teberio, F. et al.: Analytical solution for the design of planar electromagnetic bandgap structures with spurious-free frequency response. Microw. Opt. Technol. Lett., 54 (4) (2012), 956-960.

[20] Nesic, D.; Kolundzija, B.: A trapezoidal microstrip bandstop filter with efficient suppression of undesired stopbands. Optoelectron. Adv. Mater. Rapid Commun., 4 (4) (2010), 601-603.

[21] Nesic, D.A.; Kolundzija, B.M.: EBG band-stop filter with suppression of 3 spurious stop-bands, in Proc. of 6th IWAT, Article number 5464680, 2010.
[22] Nesic, D.; Kolundzija, B.: EBG Band-stop Filter with Suppression of 6 Spurious Stop-bands, in Proc. of TELSIKS 2011, Nis, 387-390.

[23] Pozar, D.M.: Microwave Engineering, 3rd ed., John Wiley and Sons, New York, 2012.

[24] Kolundzija, B.M.; Olcan, D.I.: Multiminima heuristic methods for antenna optimization. IEEE Trans. Antennas Propag., 54 (5) (2006), 1405-1415.

[25] WIPL-D software package, http://www.wipl-d.com/, WIPL-D d.o.o.: Belgrade, Serbia, 2012.

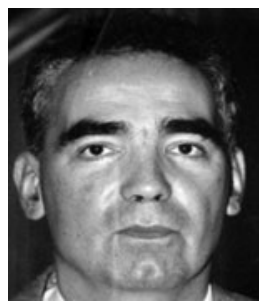

Dusan A. Nesic was born in Belgrade, Serbia, in 1961. He received the B.Sc. (1987), M.Sc. (1995), and Ph.D. (2011) degrees in electrical engineering from the School of Electrical Engineering, University of Belgrade, Serbia. He is currently with IHTM-CMT, University of Belgrade, Belgrade, Serbia. His research interests include passive microwave structures, microwave and millimeter antennas, and microwave sensors.

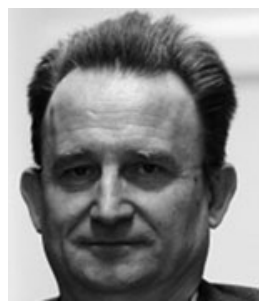

Branko M. Kolundzija was born in 1958 in Zenica, former Yugoslavia. He received the B.Sc. (1981), M.Sc. (1986), and Ph.D. (1990) degrees from University of Belgrade, Serbia. He joined the Faculty of Electrical Engineering, University of Belgrade in 1981, where he is currently a Full Professor in Electromagnetics and Antennas. He is co-author of two monographs, 29 journal articles, and 110 articles at international conferences. His research interests are electromagnetic modeling, antennas, and microwave components. He is a fellow IEEE and the main architect of the WIPL-D software.

\section{APPENDIX}

\section{REDUCTION DF FDOTPRINT AREA}

The maximum dimensions of the filters, which are $\sim 150 \mathrm{~mm}$, as shown in Fig. $7(\mathrm{a})$, can be reduced in two ways: (1) by rounding, as shown in Fig. 7 (b), and (2) by meandering as shown in Figs 7 (c) and $7(\mathrm{~d})$. Figure $7(\mathrm{e})$ shows results for corresponding $S_{21}$-parameter obtained by $3 \mathrm{D}$ EM simulation. It is seen that rounded filter has practically the same characteristics as the linear one, while meandering results in much higher loss. Namely, the excessive loss is due to bending of transmission lines. The wider a transmission line, the higher is the loss due to bending. In the case of rounded filter, the three narrowest lines are bent, while in the case of meandered filter bending is applied to six lines of different widths. Particularly, in the case of narrow meandering, the characteristic is further degraded, which can be explained by an increase of the coupling between neighboring unit cells. 